\title{
Autism assessment tools in the transition from DSM-IV to DSM-5
}

\author{
Alessandro Zuddas
}

Published online: 24 May 2013

(c) Springer-Verlag Berlin Heidelberg 2013

Autism is a heterogeneous disorder with considerable clinical diversity, aetiological heterogeneity, and multiple accompanying disorders. Conceptualization of the core autistic symptoms has evolved from the single criteria of DSM III [1] to a combination of multiple criteria in the traditional dimensions of disturbances in social relation and communication, restricted interests and repetitive behaviours of more recent classification systems. With time, empiric research leads to changes in the combination of dimensions and specific criteria: from 3 dimension with a combination of 12 criteria for autistic disorder of the DSMIV [2], the new DSM-5 [3] defines the two dimensions (social relation/communication and restricted interests/ repetitive behaviours), with 7 criteria for the "Autism spectrum disorder" (ASD). From five previously independent disorders grouped as pervasive developmental disorders (PDD) in DSM-IV, DSM-5, still provisional at the time this editorial was written, defines a single Autism spectrum disorder, and creates the new diagnostic category of Social Communication Deficit describing patients with social communication impairment without significant restricted interest or repetitive behaviours.

To manage autism phenotypic complexity and to assist the diagnostic and severity assessment, significant efforts have been devoted in developing reliable instruments able to accurately define and quantify specific autistic symptoms domains as well as behavioural and historical information from different sources. In this Issue, Falkmer and co-workers [4] systematically reviewed the accuracy,

\footnotetext{
A. Zuddas $(\square)$

Department of Biomedical Sciences, University of Cagliari and Child Neuropsychiatry, Cagliari University Hospital, Via Ospedale 119, 09124 Cagliari, Italy

e-mail: azuddas@unica.it
}

reliability, validity and utility of a series of accurately designed instruments and tools, including four observational schedules, six parent/carer interviews, two screening tools and four Asperger syndrome specific instruments, all supported by adequate validity and reliability data. They report that combined ADOS and ADI-R show the best correct classification rate for both DSM-IV autistic disorder and the more broad "Autism Spectrum Disorder" (similar to DSM-IV PDD-NOS); different versions of these instruments have been shown to be able to capture information needed to formulate the diagnosis in different ages of life, including very young children, and in patients with very different developmental levels. Many other instruments are analysed in the paper, showing that they were either lacking important information on sensitivity and specificity or did not have a large evidence-base from sufficient independent studies. Others, such as the CARS, are based mainly on clinical judgement rather than on clearly operationalized procedures.

All the instruments analysed in the paper were developed using DSM-IV constructs: interpretation of these results should consider recent papers on the use of the same diagnostic instruments in evaluating the impact of the DSM-5 criteria for the diagnosis of the "new" ASD and the need of accurate evaluation of their quantitative algorithm.

Indeed, preliminary finding suggests that a significant portion of patients currently diagnosed by DSM-IV-TR criteria may not be diagnosed by the new criteria, raising the concerns over the danger that, in many countries, these patients may lose access to services, causing significant suffering to them and their families [5].

In an epidemiological study, Mattila et al. [6] found that using the new DSM-5 criteria, only $46 \%$ of the 26 children with DSM-IV PDD (all with full scale IQ higher than 50) were identified as ASD; similar results (only $60.6 \%$ of 
DSM-IV pervasive developmental disorders also met the DSM-5 ASD diagnosis) have been reported by McPartland et al. [7]. Taheri and Perry [8] also found that only $63 \%$ of their DSM-IV PDD children met the DSM-5 ASD criteria (only $17 \%$ of the DSM-IV PDD-NOS).

On the other hand, analysing three large database including more than 5,000 children and adolescents $(4,453$ with DSM-IV PDD) Huerta et al. [9] found that most children with DSM-IV PDD diagnoses would remain eligible for an ASD diagnosis under the proposed DSM-5 criteria. Using just parent data (i.e. ADI-R), the proposed DSM-5 criteria identified $91 \%$ of children with clinical DSM-IV PDD diagnoses, with a high sensitivity rate also in specific subgroups, such as girls and children under 4 . Specificity for DSM-5 ASD and DSM-IV autistic disorder was similar (0.53), and as low as 0.23 and 0.34 for DSM-IV PDD-NOS and Asperger's disorder, respectively.

Similarly contrasting data have been reported by research groups who actually re-assessed samples of children and adolescents. In a series of studies, using a variety of different diagnostic instruments and modalities, but neither ADI-R nor ADOS, Matson and colleagues showed $32 \%$ of children [10] and $36 \%$ of adults [11] with DSMIV PDD did not meet the criteria for DSM-5 ASD. In a larger study on toddler with developmental disability, the same group measured a $47 \%$ decrease in the diagnosis of a DSM-5 ASD compared to DSM-IV PDD group (24\% for the DSM-IV autistic disorder, and $88 \%$ of the PDD-NOS) [12]. On the same sample the authors also showed that by relaxing the algorithm (i.e. decreasing to two from three the criteria needed for persistent deficit in social interaction or also decreasing to two the criteria for social deficit and to one from the two criteria needed for restrictive, repetitive pattern of behaviour or interests) the difference between the two DSMs was significantly reduced [13]. Frazier and co-workers [14], on the other hand, using caregiverreported symptoms collected with the Social Responsiveness Scale and the Social Communication Questionnaire found that DSM-5 criteria had superior specificity relative to DSM-IV-TR criteria ( 0.97 vs. 0.86 ), whereas sensitivity was lower (0.81 vs. 0.95). Requiring one less symptom criterion increased DSM-5 sensitivity (0.93 vs. 0.81 ), with minimal reduction in specificity ( 0.95 vs. 0.97$)$.

Interestingly in other two studies using ADI-R and ADOS to evaluate diagnostic information, continuity of the two DSMs was even more evident. In a sample of 489 participant with ASD (age 5-61 years, mean age 26), Mazefsky et al. [15] using combined ADOS and ADI-R ratings showed that $93 \%$ of patients met the criteria for both classification systems, whereas Barton et al. [16] applied receiver operating characteristic (ROC) curve to the combined ADI-R and ADOS scores in order to identify the best sensitivity and specificity: a 0.93 sensitivity and
0.74 specificity were obtained by applying 2 of the 3 Domain A criteria (social-communicative symptoms) and 1 point for 1 of the 4 Domain $B$ criteria (restrictive repetitive symptoms). Using ADI and ADOS to collect and evaluate information also in adults, the sensitivity of DSM-5 was significantly increased by reducing the number of criteria, without sacrificing specificity [17]. Taken together these new findings suggest that the conclusion of the Falkmer's paper may be useful also with the advent of the DSM-5 classification system.

As stated above, the significant heterogeneity in phenotypic presentation, including configuration and severity of behavioural presentation, is probably one of the main obstacles to the study of its aetiology, diagnosis treatment and prognosis [18].

The dimensional nature of ASD phenotypic presentation, combined with the need of a categorical approach for the definition of the disorder, also has a significant impact in research and translation of research findings into clinical practice. Scores of the gold standard diagnostic instruments such as ADI-R and ADOS have been also used to define empirically based behavioural phenotypes. Using a combination of latent class analysis, factorial analysis and factor mixture modelling statistical approach on the ADI-R scores of 391 newly ASD-diagnosed young children (mean age 38 months), Georgiades and co-workers [19] were able to generate a two factor (social communication deficit $S C D$ vs fixed interest and repetitive behaviour FIRB)/three classes model for the disorder. In this model, the three classes scored differently for the two factors (Class 1 higher SCD score compared to FIRB, class 2 higher FIRB scores compared to SCD, class 3 high scores in both factors), as well as on age of diagnosis and global functioning (i.e. cognitive development, language abilities and adaptive behaviour). If confirmed by further studies these classes could become severity indicators and clinical specifier, potentially useful for studying biological heterogeneity and for predicting developmental trajectories and response to treatments.

The more empirically based and more stable definition of ASD proposed by the DSM-5, perhaps with some more fine tuning in the proposed quantitative algorithm, in combination with dimensional/categorical subdivision as the proposed subgroups ("classes"), may significantly improve both clinical practice and research. It should be useful if the proposed subgroups ("classes") will be defined using, in innovative ways, the same categorical diagnostic tool such as ADI-R, ADOS or other still on developments [20].

In order to reach this goal, it is extremely needed to design several large collaborative studies. Recently, promising large European research consortia such as the Enhancing the Scientific Study of Early Autism-ESSEA 
consortium, described in this issue [21], and the Innovative Medical Initiatives project "European Autism Interventions-A Multicenter Study for Developing New Medications" EU-AIMS (http://www.eu-aims.eu) have been established and founded. This will make European research on autism spectrum disorder internationally competitive and, in turn, will improve clinical care for European children and adolescents suffering from these disorders.

\section{References}

1. American Psychiatric Association (1980) Diagnostic and statistical manual of mental disorders, 3rd edn. American Psychiatric Association, Washington, DC

2. American Psychiatric Association (2000) Diagnostic and statistical manual of mental disorders, 4th edn, Text Revision. American Psychiatric Association, Washington, DC

3. American Psychiatric Association (2012) Proposed draft revisions to DSM disorders and criteria: A 05 autism spectrum disorder. http://www.dsm5.org/ProposedRevisions/Pages/proposedrevision. aspx ?rid=94\#. Accessed 20 Dec 2012

4. Falkmer T, Anderson K, Falkmer M, Horlin C (2013) Diagnostic procedures in autism spectrum disorders; a systematic literature review. Eur Child Adolesc Psychiatry (this issue). doi:10.1007/ s00787-013-0375-0

5. Ritvo ER (2012) Postponing the proposed changes in DSM 5 for autistic spectrum disorder until new scientific evidence adequately supports them. J Autism Dev Disord 42:2021-2022

6. Mattila ML, Kielinen M, Linna SL, Jussila K, Ebeling H, Bloigu R, Joseph RM, Moilanen I (2011) Autism spectrum disorders according to DSM-IV-TR and comparison with DSM-5 draft criteria: an epidemiological study. J Am Acad Child Adolesc Psychiatry 50:58592

7. McPartland JC, Reichow B, Volkmar FR (2012) Sensitivity and specificity of proposed DSM-5 diagnostic criteria for autism spectrum disorder. J Am Acad Child Adolesc Psychiatry 2012(51):368-383

8. Taheri A, Perry A (2012) Exploring the proposed DSM-5 criteria in a clinical sample. J Autism Dev Disord 42:1810-1817

9. Huerta M, Bishop SL, Duncan A, Hus V, Lord C (2012) Application of DSM-5 criteria for autism spectrum disorder to three samples of children with DSM-IV diagnoses of pervasive developmental disorders. Am J Psychiatry 169:1056-1064

10. Worley JA, Matson JL (2012) Comparing symptoms of autism spectrum disorders using the current DSM-IV-TR diagnostic criteria and the proposed DSM-V diagnostic criteria. Res Autism Spectr Disord 6:965-970
11. Matson JL, Belva BC, Horovitz M, Bamburg J (2012) Comparing symptoms of autism spectrum disorders in a developmentally disabled adult population using the current DSM-IV-TR diagnostic criteria and the proposed DSM-5 diagnostic criteria. J Dev Phys Disabil 24:403-414

12. Matson JL, Kozlowski AM, Hattier MA, Horovitz M, Sipes M (2012) DSM-IV versus DSM-5 diagnostic criteria for toddlers with autism. Dev Neurorehabil 15:185-190

13. Matson JL, Hattier MA, Williams LW (2012) How does relaxing the algorithm for autism affect DSM V prevalence rates? J Autism Dev Disord 42:1549-1556

14. Frazier TW, Youngstrom EA, Speer L, Embacher R, Law P, Constantino J, Findling RL, Hardan AY, Eng C (2012) Validation of proposed DSM-5 criteria for autism spectrum disorder. J Am Acad Child Adolesc Psychiatry 51:28-40

15. Mazefsky CA, McPartland JC, Gastgeb HZ, Minshew NJ (2013) Brief report: comparability of DSM-IV and DSM-5 ASD research samples. J Autism Dev Disord 43:1236-1242

16. Barton ML, Robins DL, Jashar D, Brennan L, Fein D (2013) Sensitivity and specificity of proposed DSM-5 criteria for autism spectrum disorder in toddlers. J Autism Dev Disord 43:1184-1195

17. Wilson CE, Gillan N, Spain D, Robertson D, Roberts G, Murphy CM, Maltezos S, Zinkstok J, Johnston K, Dardani C, Ohlsen C, Deeley PQ, Craig M, Mendez MA, Happé F, Murphy DG (2013) Comparison of ICD-10R, DSM-IV-TR and DSM-5 in an adult autism spectrum disorder diagnostic clinic. J Autism Dev Disord (Epub ahead of print)

18. Wiggins JL, Peltier SJ, Ashinoff S, Weng SJ, Carrasco M, Welsh RC, Lord C, Monk CS (2011) Using a self-organizing map algorithm to detect age-related changes in functional connectivity during rest in autism spectrum disorders. Brain Res 1380:187-197

19. Georgiades S, Szatmari P, Boyle M, Hanna S, Duku E, Zwaigenbaum L, Bryson S, Fombonne E, Volden J, Mirenda P, Smith I, Roberts W, Vaillancourt T, Waddell C, Bennett T, Thompson A (2013) Pathways in ASD study team. Investigating phenotypic heterogeneity in children with autism spectrum disorder: a factor mixture modeling approach. J Child Psychol Psychiatry 54:206-215

20. Greaves-Lord K, Eussen ML, Verhulst FC, Minderaa RB, Mandy W, Hudziak JJ, Steenhuis MP, de Nijs PF, Hartman CA (2013). Empirically based phenotypic profiles of children with pervasive developmental disorders: interpretation in the light of the DSM-5. J Autism Dev Disord (Epub ahead of print)

21. Bölte S, Marschik PB, Falck-Ytter T, Charman T, Roeyers H, Elsabbagh M (2013) Infants at risk for autism: a European perspective on current status, challenges and opportunities. Eur Child Adolesc Psychiatry (this issue). doi:10.1007/s00787012-0368-4 